\title{
Tissue Density Mapping of Cone Beam CT Images for Accurate Dose Calculations
}

\author{
Bei Liu', Fritz A. Lerma², Jianzhou Wu ${ }^{3}$, Byong Yong $\mathrm{Yi}^{2}$, Cedric $\mathrm{Yu}^{2}$ \\ ${ }^{1}$ Department of Radiation Oncology, City of Hope Foundation, Los Angeles, USA \\ ${ }^{2}$ Department of Radiation Oncology, University of Maryland, Baltimore, USA \\ ${ }^{3}$ Department of Radiation Oncology, Swedish Cancer Institute, Seattle, USA \\ Email: beliu@coh.org
}

Received 19 February 2015; accepted 19 April 2015; published 27 April 2015

Copyright (C) 2015 by authors and Scientific Research Publishing Inc.

This work is licensed under the Creative Commons Attribution International License (CC BY). http://creativecommons.org/licenses/by/4.0/

\section{(c) (i) Open Access}

\section{Abstract}

Purpose: To improve the accuracy in megavoltage photon beam dose calculation in CBCT-based radiation treatment (RT) plans, using a kilovoltage cone-beam computed tomography (CBCT)-todensity-step (CBCT-SF) function. Materials and Methods: The CBCT-SF table is constructed from differential histograms of the voxel values of CBCT and Fan-beam CT (FBCT). From the CBCT histograms, frequency peaks representing air, lung, soft tissue and bone are observed and their widths in CT numbers are assigned to the lower and higher bounds of the steps in the CBCT-SF. The CBCT-SF is entered into a planning system as an alternative to the clinical CT-to-density table. The CT image sets studied in this work consist of FBCT and CBCT scans of three patients: a prostate cancer patient, a lung cancer patient and a head and neck patient; and of a humanoid phantom at sections of the pelvis, the thorax and the head. Deformable image registration is used to map the patient FBCT scans to the corresponding CBCT images to minimize anatomical variations. Threedimensional conformal radiotherapy (3D-CRT) and intensity-modulated radiotherapy (IMRT) plans are made on the FBCT image sets of the patients and the phantom. The plans are recalculated on the CBCT scans using both the conventional CT-to-density table and the CBCT-SF. Dose calculations on the CBCT images and FBCT images are compared using dose differences, distance to agreement (DTA), Gamma analyses and dose volume histogram (DVH) analyses. Results: The results show that IMRT plans optimized using CBCT scans and FBCT scans agree dosimetrically within $1 \%$ when the CBCT-SF is used for the CBCT-based plans, including thoracic IMRT plan. In contrast, up to $5 \%$ dose difference is observed between IMRT plans optimized on FBCT scans and CBCT scans for thoracic cases if conventional CT-to-density table is used on CBCT images. Conclusions: The simple stepwise mapping of the CBCT numbers to density using the CBCT-SF resolves the inaccuracies in dose calculations previously reported in CBCT-based RT plans. CBCT-SF can be used in ImageGuided adaptive radiotherapy planning. 


\section{Keywords}

\section{CT Number, Cone Beam CT, Re-Planning}

\section{Introduction}

Inter-fraction anatomy change may bring significant change to dose coverage for external beam radiation therapy. For example, treatment plans for prostate patients are based on fan beam computed tomography (FBCT) acquired in simulation, with full bladder and empty rectum. However, during the treatment, the patient's rectum may have a lot of gas thus bring significant anatomy change. Kilo-voltage $(\mathrm{kV})$ Cone beam computed tomography (CBCT) has been proposed for treatment re-planning [1]-[5], in image-guided adaptive radiation therapy. Adaptive plans using smaller margins for the planning target volume (PTV) based on CBCT were also investigated [6] [7]. However, due to the increased scatter radiation associated with the imaging geometry, the CT numbers of CBCT images do not have a unique, one-to-one relationship with tissue densities [8]-[11]. Using a fixed CT-to-density conversion table can lead to significant errors in heterogeneous dose calculations for RT planning.

Errors in the heterogeneous dose calculations on CBCT scans are estimated to range from $1 \%$ to $5 \%$, by comparisons to fan-beam CT (FBCT) image-based dose calculations [1] [2] [12] [13]. These differences result from inaccurate electron densities or tissue density estimates from the CBCT images used in RT planning, since these are interpolated from FBCT-to-electron density tables using CT numbers. Excessive variations in the CT numbers in a CBCT scan occur due to increased photon scattering, beam hardening effects and background variations [8]-[11]. Offline image filters or reconstruction algorithms cannot readily correct these variations [14] [15]. Because these factors are strongly dependent on the size of the imaging object, the CT numbers for tissues of the same density are also different at different anatomical sites.

To circumvent the errors in the CT numbers in CBCT scans, patient-specific 3D anatomy mapping methods have been proposed [12] [13]. In these methods the CT numbers from a planning FBCT are mapped into the CBCT anatomy using a deformable image registration platform. Thus it is assumed that the CT numbers are mapping accurately to the CBCT scan volume without actually correcting the errors in the CBCT scan directly.

Rather than using a one-to-one mapping of the CT number to tissue density, we propose an alternative method that maps a range of CT numbers to a single density value. As a result, our CBCT-to-tissue density conversion table is a step function. Because the derivation of this step function incorporates different sites in the body, this step function can be used for all sites. This paper describes the method used to derive the CBCT-to-density step function (CBCT-SF), in which the steps are preferentially selected to represent densities of air, lung, water, bone and materials of density higher than bone. The use of CBCT-SF for planning is also validated by comparing the dose calculations on CBCT images and FBCT images using planning FBCT CT number to density function, which we name it as FBCT-DF in this article.

\section{Materials and Methods}

\subsection{FBCT and CBCT Image Acquisition}

Fan Beam CT scans are acquired using a Brilliance 16-slice big-bore CT scanner (Philips Medical Systems, Cleveland, $\mathrm{OH}$ ). The CT scanner is operated in the spiral mode to acquire thoracic, pelvic and head and neck CT scans in support of RT planning. The X-ray tube is operated at $120 \mathrm{kVp}$ for the $\mathrm{HN}$ and lung scans, and at 130 $\mathrm{kVp}$ for pelvic anatomy scans. The slice thickness is $3 \mathrm{~mm}$ for thoracic and pelvic scans and $1.5 \mathrm{~mm}$ for $\mathrm{HN}$ scans. The field of view is set at $48 \mathrm{~cm}$ in all scans with 512 by 512 pixels in the axial plane.

The CBCT scans are acquired using the on-board imager (OBI) of a Trilogy linear accelerator (Varian Medical System, Palo Alto, CA), which consists of a kV X-ray source and a kV amorphous silicon flat-panel imaging detector mounted on two robotic arms. The CBCT is operated at a $150 \mathrm{~cm}$ source to detector panel distance, 125 $\mathrm{kVp}$, and $2.5 \mathrm{~mm}$ slice thickness with a 512 by 512 pixel grid reconstruction of each CT slice in the axial plane. The OBI system is calibrated to acquire images in both the full-fan mode and half-fan modes. The full fan mode uses a full bowtie and half fan mode uses a half bowtie. The CBCT scans may vary from half to full CBCT scan 
mode, based on the field of view required for imaging. Field of view dimensions greater than $26 \mathrm{~cm}$ triggers a half-fan mode, in which a half bowtie filter is used, and in which the imaging panel shifts tangentially away from the central beam axis while maintaining the same perpendicular distance to the source. Full fan mode is used for HN CBCT scanning, whereas, for thoracic site and pelvic site, the operation will default to the half-fan mode because the field of view is set to $45 \mathrm{~cm}$

The CBCT scans have a reduced scan length of $13.6 \mathrm{~cm}$, due to the acquisition limitations of the Varian OBI software and hardware. The impact of scattering dose during treatment planning is corrected by copying the top slice and the bottom slice of the CBCT scans to increase the length in the superior and the inferior directions by $10 \mathrm{~cm}$ each.

\subsection{A CBCT-to-Tissue Density-Lookup Function}

The CBCT-SF table is constructed after analyzing differential histograms of the voxel values of CBCT and FBCT image sets of various patients and phantoms. In this article, we use the tradition of CT number as used in Pinnacle treatment planning system, where CT number is 1000 unit higher than the Hounsfield unit (HU) scale. For example, HU number of water is 0 , whereas we use its CT number as 1000. Figures 1(b)-(d) plot examples of these differential histograms of CBCT and FBCT scans for HN site, pelvis site and thoracic site of three patients. Several peaks arise in the plots for both CBCT and FBCT scans: a large peak below 100 CT units appears in Figures 1(b)-(d), representing air volume; one or two peaks around 1000 CT units also appears in Figures 1(b)-(d), representing the various types of soft tissues in HN, pelvis, and thoracic sites; and a tail around 1500 CT units representing bony materials; in the thoracic site, a peak around 300 CT number representing lung tissue is also shown (Figure 1(d)). One can see that peaks in the CBCT histogram are broader than those of FBCT, especially, the double peaks around CT number of 1000 in FBCT merge to a single broad peak in CBCT, indicating that the scattering and other effects in CBCT spread out the CT number more than FBCT.

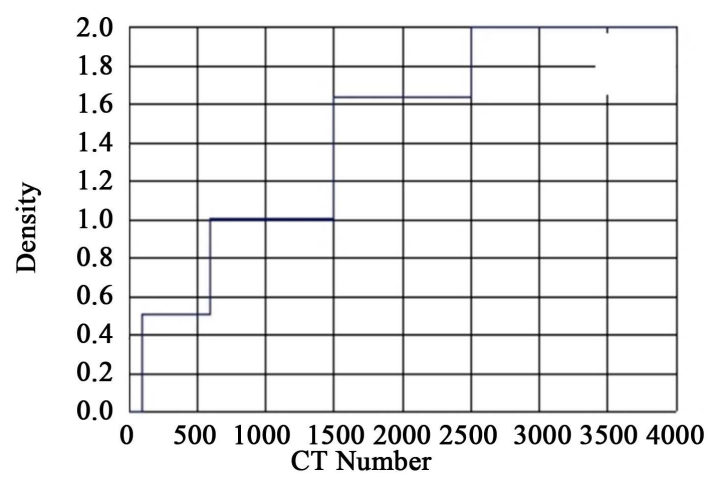

(a)

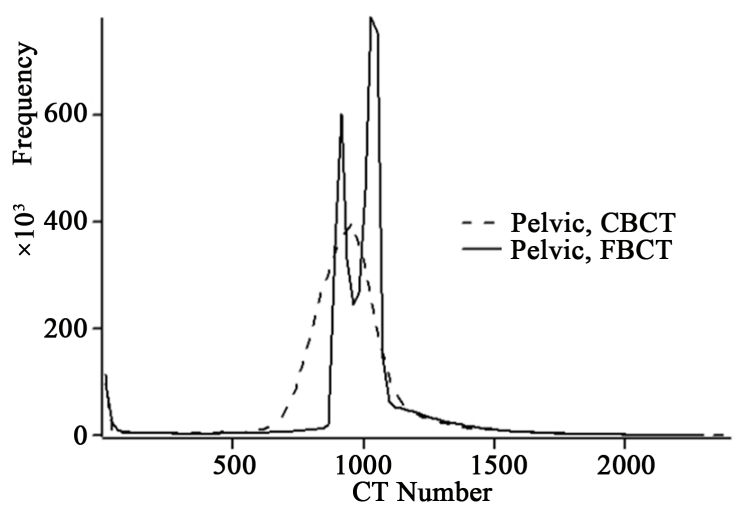

(c)

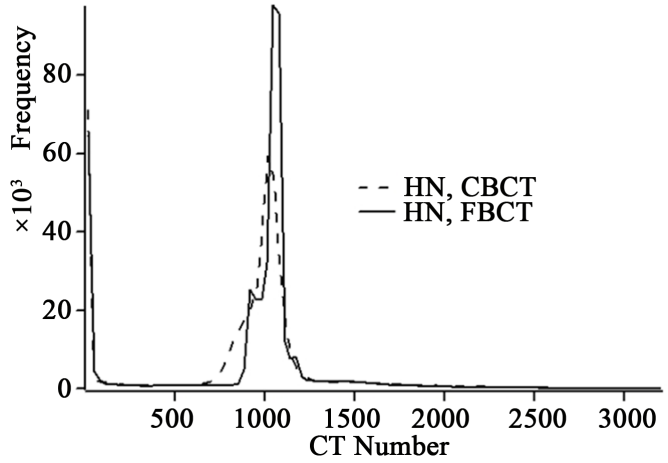

(b)

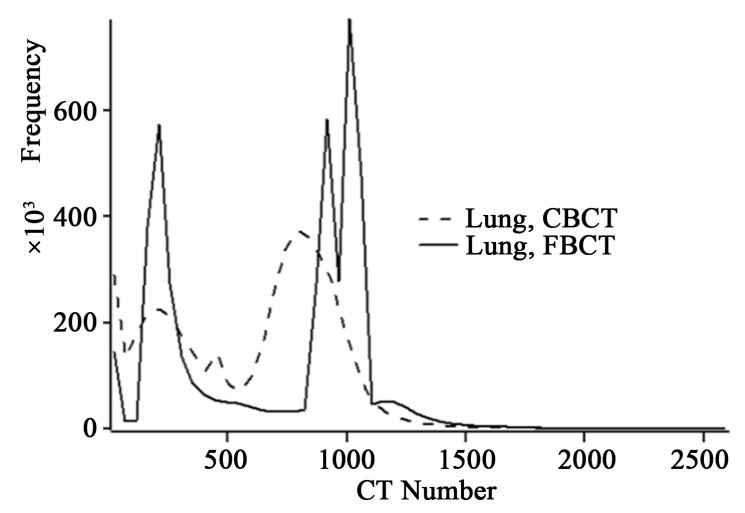

(d)

Figure 1. (a) The CBCT-SF as is entered into the CT to density interface in the treatment planning system. Comparison of the histograms of CT numbers obtained from FBCT and CBCT for patients scanned in the (b) Head and neck; (c) Pelvis; and (d) Thorax (lung). 
Based on the analysis of the differential histogram of FBCT/CBCT scans of various patients, we chose the CT number bounds for the five steps in the CBCT-SF to be: 0 - 100 for air, 101 - 600 for lung, 601 - 1500 for soft tissue, 1501 - 2500 for bone, and 2501 above for metal or equivalent material. It is easy to assign the single density values to air and soft tissue, since the air density is nearly 0 and various types of soft tissues have densities close to 1 . For bone tissue, we use the mean bone density of the patients, which is 1.63 . For lung, not only the lung densities differ in different breathing cycles, they also vary significantly for different patients [16]. In this manuscript, we look up the density corresponding to the peak CT number of lung in the differential histogram and assign it to the lung tissue. The CBCT number to density table is listed and compared with that of FBCT in Table 1. A CBCT-to-tissue density lookup function, which is a step function (CBCT-SF) as shown in Figure 1(a), is designed to map CBCT number to tissue density. The CBCT-SF contains five tissue density levels to CT numbers ranging from 0 to 4000, representing the five major materials with different densities in a human body: air, lung, soft tissue, bone, and material with higher density than bone.

\subsection{Phantom and Patient Study}

CBCT and FBCT of a solid phantoms and patients are used to evaluate the improvement of CBCT-based dose calculation using CBCT-SF vs using FBCT-DF. The solid humanoid phantom is the Rando phantom, and the phantom is scanned to simulate three cancer sites: HN, prostate and lung. Three patients with HN, prostate and lung cancers are also studied. All patient images in this study were evaluated in offline analyses, following patient consent to use these images for research purposes. Patients may have received multiple CBCT scans but only the first CBCT scan is used in this study.

The advantage of using rigid-body Rando phantom in this study is that rigid-body registration can align the CBCT images with the FBCT scans accurately, therefore the dose difference calculated between FBCT and CBCT only comes from the CT number difference between FBCT and CBCT. In treatment planning, regions of interest (ROIs) are contoured on the FBCT scans. The ROI's are then mapped to the corresponding CBCT scans after a rigid-body registration between CBCT and FBCT. The registration is performed using the Syntegra package in Pinnalce 8.1v (Philips Medical Systems, Cleveland, OH).

For patient study, the FBCT and CBCT were acquired on different days, thus exhibit anatomical variation due to organ deformation. Consequently, CBCT and FBCT images are likely to exhibit differences that are not solely due to CT number deviations, and this problem can be solved using deformable registration.

A deformable registration is applied to map the FBCT scans to the CBCT scans, using a B-Spline imagedensity based algorithm to correct for such extraneous deviation [18]. The deformable registration is conducted

Table 1. The clinical CT (FBCT) to tissue density lookup table used in our clinical treatment plans on FBCT scans, and the CBCT-to-density table (CBCT-SF) implemented in this work are presented. Notably, the CBCT-to-density table introduces steps of isodensity over wide ranges of CT Number.

\begin{tabular}{cccc}
\hline Clinical CT-to-density table for $120 \mathrm{kVp}$ and $130 \mathrm{kVp}$ scans & \multicolumn{2}{c}{ CBCT-to-density step function (CBCT-S Function) } \\
\hline CT Number & Density $\left(\mathrm{g} / \mathrm{cm}^{3}\right)$ & CT Number & Density $\left(\mathrm{g} / \mathrm{cm}^{3}\right)$ \\
\hline 0 & 0.000 & 0 & 0.000 \\
31 & 0.001 & 100 & 0.000 \\
281 & 0.450 & 101 & Lung density \\
902 & 0.920 & 600 & 1.000 \\
1003 & 1.000 & 601 & 1.000 \\
1233 & 1.122 & 1500 & 1.630 \\
2282 & 1.820 & 1501 & 1.630 \\
& & 2500 & 2.000 \\
\hline
\end{tabular}


with an imaging tool kit software platform on a linux PC system, where a B-Spline deformable registration is implemented as shown by Rueckert et al. [18]. The deformed FBCT scan, instead of the raw FBCT scans, are loaded into the treatment planning system and segmented manually. The contours are then aligned to the CBCT scan with no further deformation. Therefore, the deformed FBCT images and CBCT images contain the same contours for the respective plan comparisons.

Treatment plans on the Rando Phantom includes: a single AP beam incident on the lung, an SBRT plan with 12 coplanar beams incident on a fictitious target near mediastinum, a prostate IMRT plan and an HN IMRT plan. For the non-IMRT plans, three dose calculations are performed and compared: 1) calculation based on FBCT and 2) on CBCT using FBCT-DF, 3) calculation based on CBCT using CBCT-SF. The CBCT-based plan doses are aligned to the doses on the FBCT and are then compared using distance to agreement (DTA) and gamma analyses [17]. The gamma analyses are made on 2D planes, using $2 \%$ dose difference and $2 \mathrm{~mm}$ DTA; DTA evaluation uses a $2 \mathrm{~mm}$ criterion, and the percent dose difference uses a $2 \%$ criterion. The results are expressed as the percentage of points passing the corresponding criterion in each test. For IMRT plans, the plans are optimized on the FBCT, and then the optimized plans are copied to the corresponding CBCT scan following rigidbody alignment and re-calculated on CBCT without re-optimization, using FBCT-DF and CBCT-SF, respectively. The dose distributions calculated on CBCT are then compared to the dose calculated on FBCT point by point, in terms of dose difference, DTA and gamma analysis.

For patient study, only IMRT plans for HN, prostate and lung cancers are studied. Unlike the phantom study, where the IMRT plans are optimized on FBCT and re-calculated on CBCT with no re-optimization, IMRT plans on patient are optimized on deformed FBCT, on CBCT using FBCT-DF, and on CBCT using CBCT-SF, respectively. In the IMRT optimization, we use the same optimization parameters and the beams are reset to open beams so that the difference in the optimization path based on FBCT and CBCT comes only from their difference in CT numbers. The DVH of these plans are also compared.

For both the phantom study and patient study, the IMRT beam setups are the same. The HN IMRT plans use five coplanar $6 \mathrm{MV}$ beams at gantry angles of $0^{\circ}, 160^{\circ}, 220^{\circ}, 260^{\circ}$, and $310^{\circ}$, with an $8 \mathrm{~mm}$ margin PTV, and with cord, and parotids as avoidance structures. The prostate IMRT plan uses seven coplanar 6 MV beams at gantry angles of $0^{\circ}, 50^{\circ}, 100^{\circ}, 150^{\circ}, 210^{\circ}, 260^{\circ}$ and $310^{\circ}$, and it covers a PTV resulting from an isotropic $5 \mathrm{~mm}$ margin from the prostate contour, with the sparing structures being rectum and bladder. The IMRT plan for lung cancer uses the same beam setup as the prostate IMRT, with a $10 \mathrm{~mm}$ margin PTV, and with heart, normal lung tissue and cord as avoidance structures. The prescription is 180 cGy per fraction for HN and prostate cancer, and 200 cGy per fraction for lung cancer.

\section{Results}

\subsection{Phantom Study}

Dose difference, DTA and gamma analysis are conducted to compare the dose calculations based on CBCT using FBCT-DF and dose calculations based on FBCT (hollow symbol in Figure 2), as well as to compare the dose calculations based on CBCT using CBCT-SF and dose calculated from FBCT (solid symbols in Figure 2). The comparisons above are applied to the axial slices that passing through the iso center for points/voxels that receive at least $20 \%$ of the maximum dose. In Figure 2, the Y coordinates describe the percentage of points that pass the test criteria, which are $2 \%$ for dose difference, $2 \mathrm{~mm}$ for DTA, and $2 \%$ dose difference and $2 \mathrm{~mm}$ DTA for gamma analysis; the $\mathrm{X}$ coordinates describe the treatment planning sites and methods. This figure represent pass rate of gamma analyses as squares, passing rate of DTA as upward triangles, and passing rate of dose-difference as downward triangles. In this figure, one can see that for pelvis IMRT, lung single beam and lung SBRT plans, the solid symbols exhibit significantly higher passing rate than hollow symbols, indicating that using CBCT-SF achieve a significantly better accuracy than using FBCT-DF for CBCT-based planning and dose calculations. Especially, the solid squares all have values of more than $97 \%$ of passing rate for gamma analysis, indicate the excellent agreement between doses calculated from CBCT using CBCT-SF and doses calculated from FBCT.

However, for the HN IMRT, the improvement of CBCT-based dose calculation accuracy using CBCT-SF is not that much better than using FBCT-DF. The passing rates of gamma and dose difference are essentially the same as shown in Figure 2, indicating, for HN site, that CBCT-based dose calculation using either CBCT-SF or FBCT-DF agree excellently with doses calculated from FBCT. This is possibly due to the fact that head is small, 


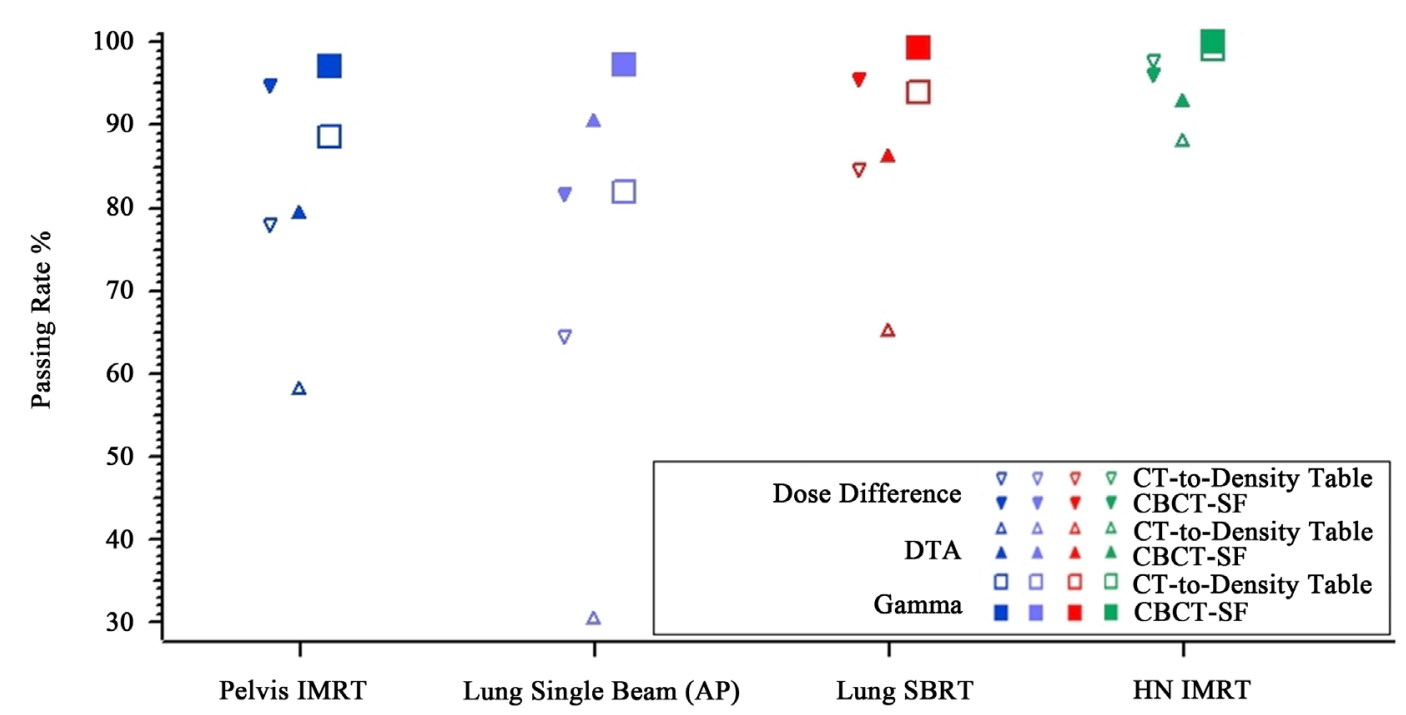

Figure 2. The percent passing rate of Gamma-, DTA-, and dose-difference analyses are given for four plans computed on FBCT and CBCT of the Rando phantom. The analyses compare dose calculations on FBCT and CBCT using the FBCT-DF with open symbols. Comparison between FBCT based-planning using FBCT-DF and CBCT-based planning using CBCT-SF are also shown by solid symbols. Dose difference is shown with triangles pointing downwards, DTA is shown with triangles point upwards and Gamma analyses are shown with squares.

thus the increase of photon scattering and beam hardening effects are not as prominent as other sites such as pelvis and thorax. One can observe this from Figure 1(b): the broadening of the CBCT's histogram peak around CT number 1000 for HN is much less prominent than those for pelvis and lung. Therefore, CBCT's CT number is closer to FBCT's CT number, compared to pelvic and thoracic sites, so using CBCT-SF provides no significant advantage than using FBCT-DF for CBCT-based treatment planning of HN cancers.

Percent dose differences between FBCT-based SBRT plan and CBCT-based SBRT plan are overlaid on the CBCT scan of the Rando phantom thoracic section in Figure 3, where Figure 3(a) uses FBCT-DF and Figure 3(b) uses CBCT-SF for CBCT-based SBRT plans. Clearly, one can see that the region around iso center is surrounded by the red-3.0\% dose difference line in Figure 3(a), whereas it is surrounded by yellow $0.0 \%$ dose difference line in Figure 3(b), indicating local percent dose differences decrease from over 3\% deviation to less than 1\% deviation after CBCT-SF replaces FBCT-DF in CBCT-based treatment planning.

As addressed earlier, the single density value used to represent lung tissue is chosen to be the density corresponding to the peak CT number in the differential histogram of lung, which is $0.5 \mathrm{~g} / \mathrm{cm}^{3}$ in this study. However, human lung densities have large variability that ranges from 0.3 to $0.7 \mathrm{~g} / \mathrm{cm}^{3}$. To investigate the sensitivity of dose calculation to this single density value, we assign the single lung density value from 0 to $1 \mathrm{~g} / \mathrm{cm}^{3}$ in the CBCT-SF and use it to re-compute the SBRT plan on the CBCT scan of the humanoid thorax phantom, and compare the plan with FBCT-based plan. Figure 4 plots the passing rate of dose difference, DTA and gamma vs. the single density value of lung. A density plateau ranging from $0.4-0.6 \mathrm{~g} / \mathrm{cm}^{3}$ has nearly $100 \%$ passing rate for gamma, indicating that the single density value in the CBCT-SF can be assigned a value in the range of peak lung density $\pm 0.1 \mathrm{~g} / \mathrm{cm}^{3}$ without significant accuracy loss of dose calculation.

\subsection{Patient Study}

Figure 5 compares the DVH curves of IMRT plans optimized independently on deformed FBCT using FBCT-DF (solid lines), on CBCT using FBCT-DF (dashed lines) and on CBCT using FBCT-SF (dotted lines). The IMRT beam setup, optimization parameters and constraints are the same for the three methods. Figures 5(a)-(c) are the DVHs for localized prostate cancer, nasopharynx cancer and lung cancer, respectively. One can see, for prostate cancer in Figure 5(a), that the dotted curves almost coincident with the solid curves, whereas the dashed curves of PTV and rectum are clearly off from the corresponding solid curves. For lung cancer in Figure 5(c), dotted curves are very close to the solid curves, while the dashed curve of PTV is significantly off 


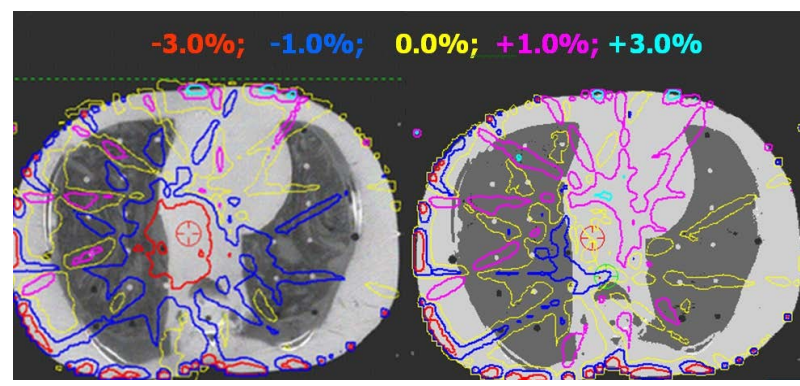

Figure 3. (a) The percent dose-difference of an SBRT plan computed on FBCT using FBCT-DF and on CBCT using FBCT-DF is overlayed on an axial plane from the CBCT image of a humanoid thorax section phantom; (b) The percent dose-difference between SBRT plans computed on FBCT using FBCT-DF and on CBCT using the CBCT-SF.

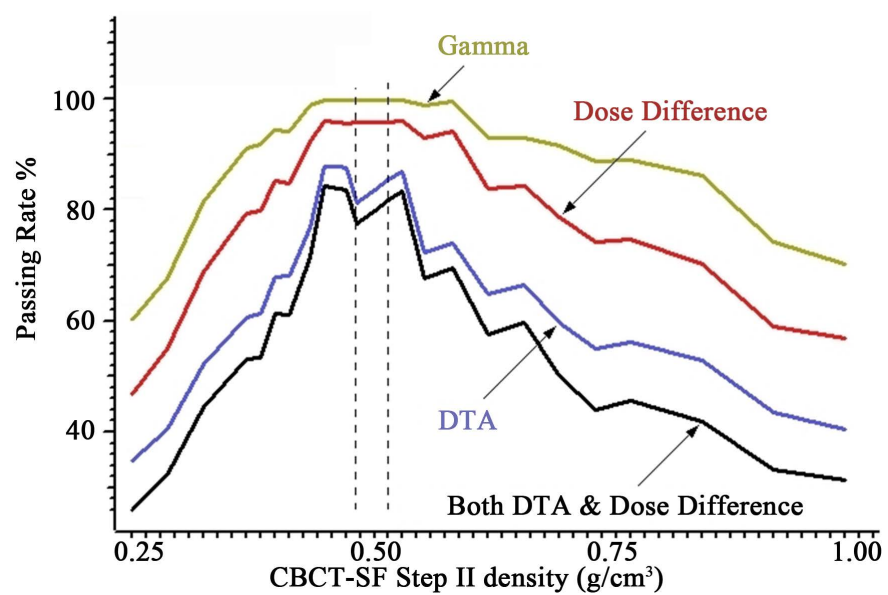

Figure 4. The gamma-, dose-difference-, and DTA-passing rate between the FBCTbased SBRT plan using FBCT-DF and the CBCT-based SBRT plan using CBCT$\mathrm{SF}$, varying the single density value of lung in CBCT-SF, which is the second step in the CBCT-SF of Figure 1(a).

from the solid curve of PTV. Therefore, for both pelvic and thoracic IMRT plans, CBCT-based IMRT plans using CBCT-SF are in better agreement with the FBCT-based optimizations. For the IMRT HN plans shown in Figure 5(c), both CBCT-based IMRT plans agree with the FBCT-based plans excellently, suggesting a lesser need for the CBCT-SF for HN planning.

\section{Discussion}

Planning fan beam CT-to-density tables, FBCT-DF, are monotonic functions used to estimate material electron densities [19] [20]. The bias and variability of CBCT's CT number introduced by increased scattering, beam hardening effect and etc. may introduce significant dose calculation errors when FBCT-DF is used for CBCTbased RT planning. The ideal way to solve this problem is to use a CT-to-density table specifically made for CBCT. However, this is impractical [1] [8] due to the variability in CT numbers of a CBCT scan. For example, the same type of tissue in different anatomic sites may have different CT number in CBCT, because tissue's CT numbers depend on the size of the imaging object. The variability in CT numbers directly impacts the accuracy of dose calculation. The CBCT-SF, on the other hand, minimizes the uncertainty in the relation of the CT numbers and the densities by assigning a single density value to a range of CT numbers. In most anatomic sites except thoracic site, air and soft tissue, which respectively have a density of 0 and close to unit no matter what imaging method is used, are the majority materials that treatment beams pass through.

Within a specific patient, the variability of lung tissue density is comparable to that of soft tissue, as can be seen from the spread of the corresponding peaks of lung and soft tissues in Figure 1(d). Our results show, like 


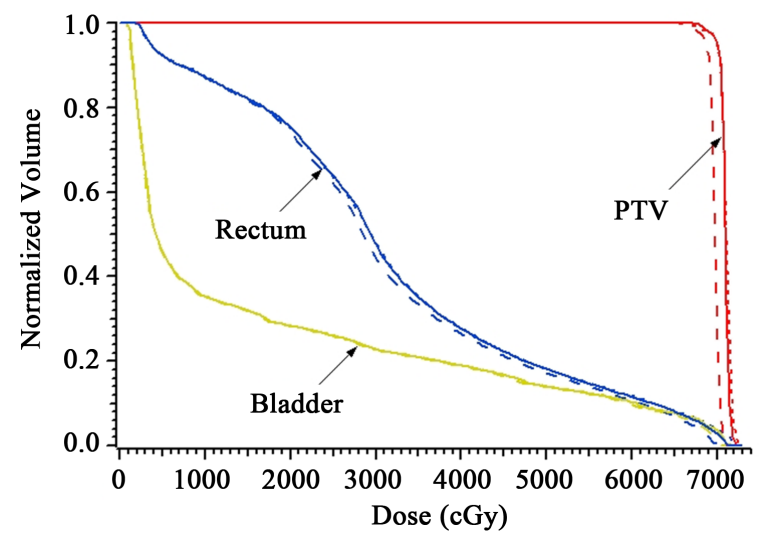

(a)

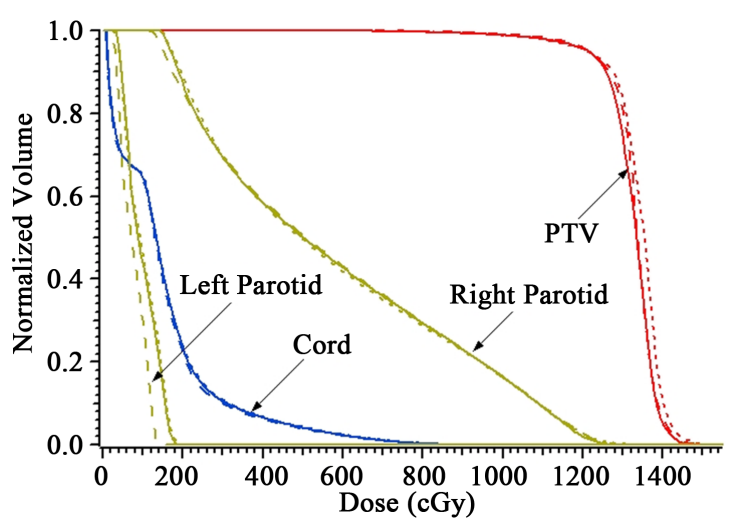

(b)

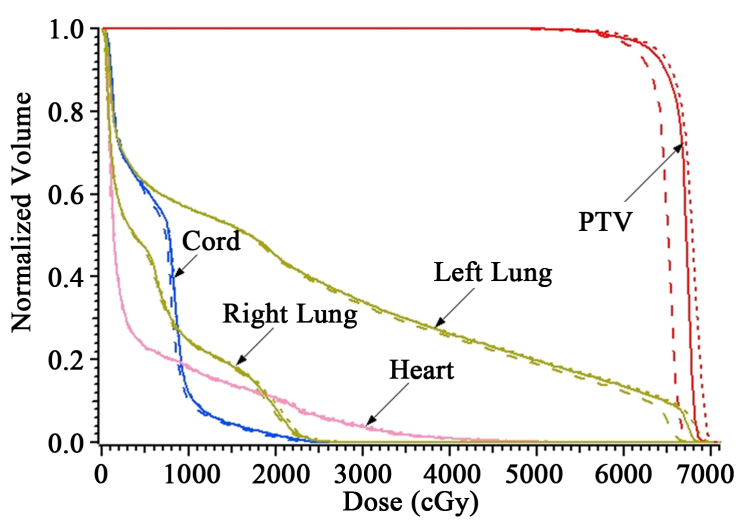

(c)

Figure 5. The DVHs of (a) pelvic; (b) head and neck and (c) lung IMRT plans are shown. Each histogram contains three treatment plan calculations: solid lines for FBCT-based plans using FBCT-DF, dashed lines for CBCT-based plans using FBCT-DF; and dotted lines for CBCT-based plans using CBCT-SF.

the soft tissues, that lung tissue densities can be represented by a single value to achieve excellent accuracy of dose calculation in CBCT-based planning. However, among different patients, lung tissue density varies significantly [8] [16], ranging from $0.3-0.7 \mathrm{~g} / \mathrm{cm}^{3}$. Our result (Figure 4) shows that single density value of lung in CBCT-SF can be off by $\pm 0.1 \mathrm{~g} / \mathrm{cm}^{3}$ from the lung density in order to keep excellent accuracy of dose calculation. Therefore, there is no single density value of lung in CBCT-SF can be applied to the treatment planning of all lung cases. On the contrary, several CBCT-SFs are needed for thoracic treatment planning according the patients' lung density, for example, one can design three CBCT-SFs by assigning lung density to be $0.35,0.50$ and 0.65 $\mathrm{g} / \mathrm{cm}^{3}$ and uses them accordingly.

CBCT-SF based treatment planning dose calculations are especially notable in the vicinity of lung, where IMRT plans optimized on CBCT images exhibit less than 1\% dose differences compared to those plans using FBCT images. These results also show that small tissue density variations may be ignored since these have a negligible impact on megavoltage photon beam dose calculation accuracy. We have shown that by the CBCT$\mathrm{SF}$, a CBCT image converts into a 5 density-level 3D map for dose calculation purposes, and that this map can bring a $1 \%$ agreement with calculations using FBCT scans. Notably, CT-to-density tables are commissioned for clinical use to achieve 1\% - 3\% agreement between RT planning dose calculations and chamber measurements in a phantom.

\section{Conclusion}

Using a histogram mapping strategy, we developed a novel method to map CBCT numbers to tissue densities, using a CBCT-to-density step function (CBCT-SF). This CBCT-SF achieves CBCT-based treatment planning dose calculations and IMRT optimizations that are interchangeable with those using FBCT scans. The CBCT-SF 
can be readily entered into a radiation treatment planning system without introducing spurious errors in dose calculations. Thus, the CBCT-SF is a practical solution that may be readily implemented in CBCT-based image guided adaptive radiotherapy planning [1] [2] [12] [13] [21] [22].

\section{References}

[1] Yoo, S. and Yin, F.F. (2006) Dosimetric Feasibility of Cone-Beam CT-Based Treatment Planning Compared to CT-Based Treatment Planning. International Journal of Radiation Oncology*Biology* Physics, 66, 1553-1561. http://dx.doi.org/10.1016/j.ijrobp.2006.08.031

[2] Yang, Y, Schreibmann, E., Li, T., Wang, C. and Xing, L. (2007) Evaluation of On-Board kV Cone Beam CT (CBCT)Based Dose Calculation. Physics in Medicine \& Biology, 52, 685-705. http://dx.doi.org/10.1088/0031-9155/52/3/011

[3] Ding, X., Duggan, D.M., Coffey, C.W., Deeley, M., Hallahan, D.E., Cmelak, A. and Malcolm, A. (2007) A Study on Adaptive IMRT Treatment Planning Using KV Cone-Beam CT. Radiotherapy \& Oncology, 85, 116-125. http://dx.doi.org/10.1016/j.radonc.2007.06.015

[4] Hatton, J., McCurdy, B. and Greer, P.B. (2009) Cone Beam Computerized Tomography: The Effect of Calibration of the Hounsfield Unit Number to Electron Density on Dose Calculation Accuracy for Adaptive Radiation Therapy. Physics in Medicine \& Biology, 54, N329-N346. http://dx.doi.org/10.1088/0031-9155/54/15/N01

[5] Li, J., Yao, W., Xiao, Y. and Yu, Y. (2013) Feasibility of Improving Cone-Beam CT Number Consistency Using a Scatter Correction Algorithm. Journal of Applied Clinical Medical Physics, 14, 167-176.

[6] Hawkins, M.A., Brooks, C., Hansen, V.N., Aitken, A. and Tait, D.M. (2010) Cone Beam Computed Tomography-Derived Adaptive Radiotherapy for Radical Treatment of Esophageal Cancer. International Journal of Radiation Oncology*Biology* Physics, 77, 378-383. http://dx.doi.org/10.1016/j.ijrobp.2009.05.045

[7] Foroudi, F., Wong, J., Kron, T., Rolfo, A., Haworth, A., Roxby, P., Thomas, J., Herschtal, A., Pham, D., Williams, S., Tai, K.H. and Duchesne, G. (2011) Online Adaptive Radiotherapy for Muscle-Invasive Bladder Cancer: Results of Pilot Study. International Journal of Radiation Oncology*Biology* Physics, 81, 765-771. http://dx.doi.org/10.1016/j.ijrobp.2010.06.061

[8] Endo, M., Tsunoo, T. and Nakamori, N. (2001) Effects of Scatter Radiation on Image Noise in Cone Beam CT. Medical Physics, 28, 469-474. http://dx.doi.org/10.1118/1.1357457

[9] Hsieh, J., Molthen, R., Dawson, C. and Johnson, R. (2000) An Iterative Approach to the Beam Hardening Correction in Cone Beam CT. Medical Physics, 27, 23-29. http://dx.doi.org/10.1118/1.598853

[10] Siewerdsen, J.H., Daly, M.J., Bakhtiar, B., Moseley, D.J., Richard, S., Keller, H. and Jaffray, D.A. (2005) A Simple, Direct Method for x-Ray Scatter Estimation and Correction in Digital Radiography and Cone-Beam CT. Medical Physics, 33, 187-197. http://dx.doi.org/10.1118/1.2148916

[11] Siewerdsen, J.H. and Jaffray, D.A. (2001) Cone-Beam Computed Tomography with a Flat-Panel Imager: Magnitude and Effects of X-Ray Scatter. Medical Physics, 28, 220-231. http://dx.doi.org/10.1118/1.1339879

[12] Zijtveld, M.V., Dirkx, M. and Heijmen, B. (2007) Correction of Conebeam CT Values Using a Planning CT for Derivation of the "Dose of the Day". Radiotherapy and Oncology, 85, 195-200. http://dx.doi.org/10.1016/j.radonc.2007.08.010

[13] Yan, D., Wong, J., Vicini, F., Michalski, J., Pan, C., Frazier, A., Horwitz, E. and Martinez, A. (1997) Adaptive Modification of Treatment Planning to Minimize the Deleterious Effects of Treatment Setup Errors. International Journal of Radiation Oncology*Biology*Physics, 38, 197-206. http://dx.doi.org/10.1016/S0360-3016(97)00229-0

[14] Zhang, Y., Zhang, L., Zhu, X.R., Lee, A.K., Chambers, M. and Dong, L. (2007) Reducing Metal Artifacts in Cone-Beam CT Images by Preprocessing Projection Data. International Journal of Radiation Oncology*Biology* Physics, 67, 924-932. http://dx.doi.org/10.1016/j.jjrobp.2006.09.045

[15] Manzke, R., Grass, M. and Hawkes, D. (2004) Artifact Analysis and Reconstruction Improvement in Helical Cardiac Cone Beam CT. IEEE Transactions on Medical Imaging, 23, 1150-1164. http://dx.doi.org/10.1109/TMI.2004.830804

[16] Van Dyk, J., Keane, T.J. and Rider, W.D. (1982) Lung Density as Measured by Computerized Tomography: Implications for Radiotherapy. International Journal of Radiation Oncology*Biology*Physics, 8, 1362-1372. http://dx.doi.org/10.1016/0360-3016(82)90587-9

[17] Low, D.A., Harms, W.B., Mutic, S. and Purdy, J.A. (1998) A Technique for the Quantitative Evaluation of Dose Distributions. Medical Physics, 25, 656-661. http://dx.doi.org/10.1118/1.598248

[18] Rueckert, D., Sonoda, L.I., Hayes, C., Hill, D.L., Leach, M.O. and Hawkes, D.J. (1999) Nonrigid Registration Using Free-Form Deformations: Application to Breast MR Images. IEEE Transactions on Medical Imaging, 18, 712-721. http://dx.doi.org/10.1109/42.796284

[19] Schneider, U., Pedroni, E. and Lomax, A. (1996) The Calibration of CT Hounsfield Units for Radiotherapy Treatment 
Planning. Physics in Medicine and Biology, 41, 111-124. http://dx.doi.org/10.1088/0031-9155/41/1/009

[20] Chu, J.C.H., Ni, B., Kriz, R. and Saxena, A. (2000) Applications of Simulator Computed Tomography Number for Photon Dose Calculations during Radiotherapy Treatment Planning. Radiotherapy and Oncology, 55, 65-73. http://dx.doi.org/10.1016/S0167-8140(00)00159-6

[21] Mohan, R., Zhang, X., Wang, H., Kang, Y., Wang, X., Liu, H., Ang, K.K., Kuban, D. and Dong, L. (2005) Use of Deformed Intensity Distributions for Online Modification of Image-Guided IMRT to Account for Interfractional Anatomic Changes. International Journal of Radiation Oncology*Biology*Physics, 61, 1258-1266. http://dx.doi.org/10.1016/j.ijrobp.2004.11.033

[22] Ahunbay, E., Chen, G., Peng, C., Yu, C., Narayanan, S., Lawton, C. and Li, X.A. (2007) An On-Line Adaptive Planning Strategy for Inter-Fraction Image Guidance. International Journal of Radiation Oncology*Biology*Physics, 69, S23. http://dx.doi.org/10.1016/j.ijrobp.2007.07.040 